\title{
An innovative demand side management for vulnerable hybrid microgrid
}

\begin{abstract}
The future of power system will be highly influenced by Microgrid and Smart Grid with renewable energy resources. A standalone Microgrid suffer by intermittent generation and lack of supply due to harsh environment condition. Demand Side Management (DSM) manipulates demand instead of generation to increase energy efficiency. The existing DSM is developed by utilities including financial incentive plan, load efficiency and etc. to improve load factor. These plans are required adjustment and improvement to meet vulnerable standalone system with limited source. Interactive demand responses with SMS and direct access to individual load are introduced for DSM. This work intentionally shows a vulnerable Microgrid system with limited source to highlight the DSM function. DSM controller modeled and simulated in Simulink. The proposed DSM module, isolate the loads based on predefined load priority. Different scenarios for generation units are simulated to test DSM action in different situation. It will reduce unnecessary investment to generation unit expansion and will improve load factor.
\end{abstract}

Keyword: Demand response (DR); Demand side management (DSM); Hybrid system; Microgrid; Smart grid 